\title{
Implementation of metadata logging and power loss recovery for page-mapping FTL
}

\author{
Seung-Ho Lim ${ }^{\text {a) }}$ \\ ${ }^{1}$ Hankuk University of Foreign Studies, 89 Wangsan-ri, Mohyeon-Myeon, Yongin-si, \\ Korea \\ a)slim@hufs.ac.kr
}

\begin{abstract}
In NAND flash systems, the mapping table and related metadata information of FTL should be recovered from sudden power loss to provide data consistency. Nowadays, a lot of FTLs have been reported, however, there are little development reports for FTL recovery schemes. In this paper, we design fast metadata logging and recovery scheme to support efficient and fast power loss recovery. The designed recovery scheme is based on the page-level mapping FTL. In the designed FTL, All the metadata changes are logged into dedicated flash block, which minimize the frequency of metadata updates. At the recovery stage, only metadata blocks are retrieved. The designed FTL metadata logging and recovery scheme support fast sudden power loss recovery, with small amount of metadata logging overhead.
\end{abstract}

Keywords: NAND flash, FTL, metadata logging, PLR

Classification: Storage technology

\section{References}

[1] A. Ban: U.S. Patent 5,937,425 (1997).

[2] S. Lee, S. Jung and Y. H. Song: IEEE Int. Conf. Network Infrastructure and Digital Content (2010) 264.

[3] T.-S. Chung, M. Lee, Y. Ryu and K. Lee: J. Systems Architecture 54 (2008) 935.

[4] S. Moon, S.-P. Lim, D.-J. Park and S.-W. Lee: 8th IFIP WG 10.2 Int. Conf. Software Technologies for Embedded and Ubiquitous Systems (2010) 13.

[5] C.-H. Wu and H.-H. Lin: ACM Transactions on Design Automation of Electronic Systems 17 (2012) 14.

[6] Samsung Electronics: Nand flash-memory datasheet (2011) http://www.datasheetcatalog.com/samsungelectronic/41/ 


\section{Introduction}

In NAND flash memory, read and write commands are performed in units of page. The erase operation is performed in units of block, whose size is much larger than that of pages. To overcome the mismatch between write and erase operations, Flash Translation Layer (FTL) [1], has been developed. The role of FTL is address translation between logical address of file system and physical address of flash memory itself. FTL performs out-of-place updates which in turn help to hide the erase operation. When the number of free pages is insufficient for write operations, free pages should be made by garbage collection (GC), where GC is the process that makes available free region by selecting one block, moving data of valid pages to other region, and erasing the block. Storage systems should prepare sudden power loss recovery (PLR) method to preserve data consistency and data integrity. In case of NAND, the mapping table and related metadata information for the FTL should be recovered from sudden power loss to provide proper operations. The efficiency of PLR is dependent on the FTL's metadata architecture. In general, there are three types of FTL mapping method; block-level and page level and mixture of these two. For the block-level and mixture of block and page mapping management, previous research for PLR methods [3, 4] was developed. However, there are little previous research works published for power loss recovery for page-level FTL. The spare-map-based recovery schemes can be applied to page level FTL $[2,5]$, which means that the mapping information are stored in the spare region in Flash, and these are scanned throughout the all area during recovery time. This is good for runtime overhead since there is little metadata update overhead during runtime. However, it reveals critical scalability problem as NAND flash capacity grows, since recovery time is getting longer as the capacity grows.

In this paper, we design efficient metadata architecture and logging scheme to support efficient and fast power loss recovery, with minimizing runtime overhead. The designed recovery scheme is based on the page-level mapping FTL. In our design, the metadata includes, Root block, BlockMap block, PageMap block, and MapLog block. All the metadata changes for the current data block are aggregated in main memory, called MapLogInfo, and the metadata changes are logged into one page of dedicated flash block, called MapLog. The logging scheme can minimize metadata update overhead, which is negligible in comparison with overall data write operations. At the recovery time, only metadata blocks are required to be retrieved, the scanned region is also negligible in comparison with data region.

\section{FTL power loss recovery}

The designed FTL metadata architecture is shown in Figure 1. In the figure, the Root block contains block numbers for metadata blocks (i.e., BlockMap block, PageMap block, and MapLog block). The block 0 and 1 are used for pivot Root block. At first, block 0 is assigned to Root block. In the last updated page in the Root block, RootInfo[] is stored with the locations of meta 


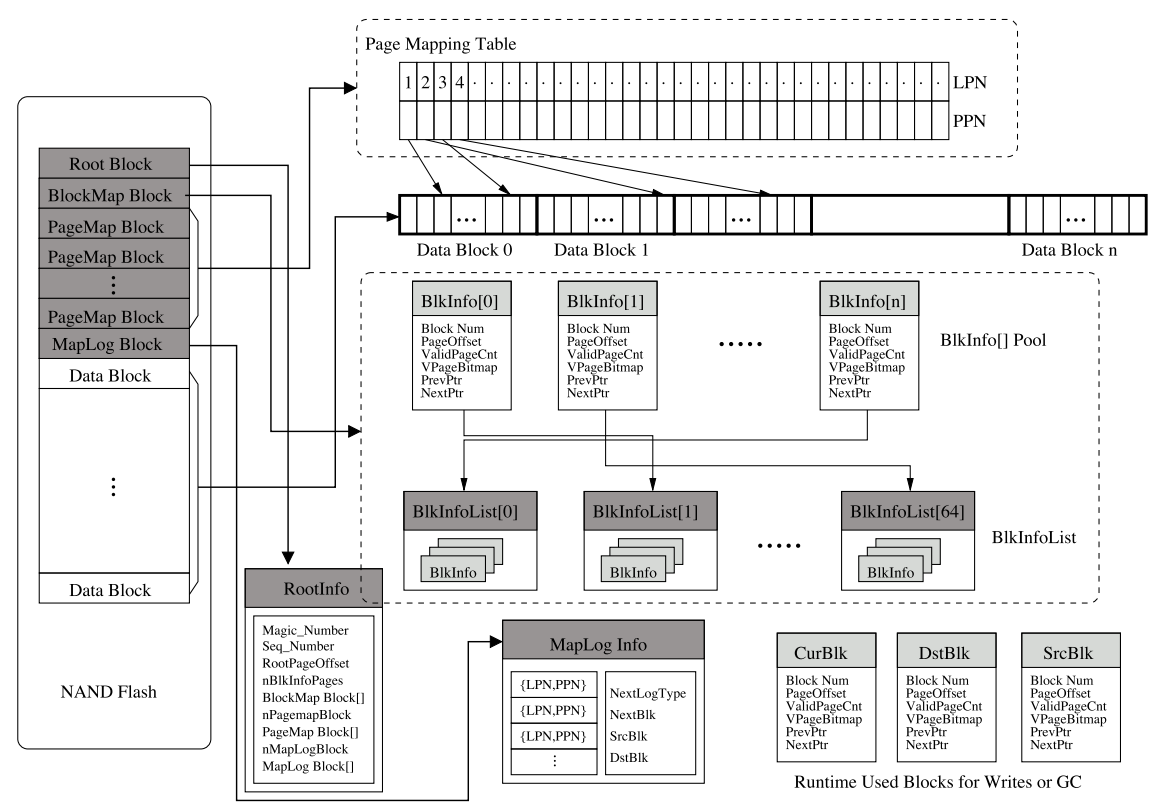

Fig. 1. Runtime FTL Metadata structure in-main memory and snapshot of Metadata in-NAND Flash.

blocks information. Whenever the meta blocks are changed, the RootInfo is updated as a log manner in the Root block. When the update reaches to last page of current Root block, i.e, block 0, the Root block is changed to block 1, and the update is continued. The BlockMap block contains usage of each data blocks and additional block's list information. There are two data structures for representing Block usage, i.e., BlkInfo[], and BlkInfoList[]. The BlkInfo[] array contains status information for each data blocks. The BlkInfoList[] is hash-like data structure that has 65 buckets from 0 to 64 , if there are 64 pages in on block. Each bucket has doubled-linked list of blocks that have same valid page count. The BlkInfoList[0] bucket contains free blocks, and BlkInfolist[64] bucket contains blocks with full valid pages. The PageMap blocks contains page mapping table information. Each entry of page mapping table is a pair of Logical Page Number (LPN) and Physical Page Number (PPN). The last part of metadata is MapLog block. While in-memory metadata contains up-to-date metadata status, in-NAND meta blocks contains snapshot status at some time stamp. The changes between in-main memory and in-NAND metadata are stored in MapLog block.

In our design, the in-NAND flash meta blocks contains the snapshot status of metadata, which means in-NAND flash meta blocks contains metadata information at some time stamp. On the other hand, in-main memory metadata architecture contains up-to-date metadata information, which is different from snapshot of in-NAND flash meta blocks. Whenever write occurs, the mapping information should be changed since data are written to new clean pages. To preserve up-to-date state of metadata, the changes is directly applied to in-memory metadata architecture, however, in-NAND metadata are not directly updated, since it makes huge overhead of flash operations. The differences between in-NAND and in-memory are generated 


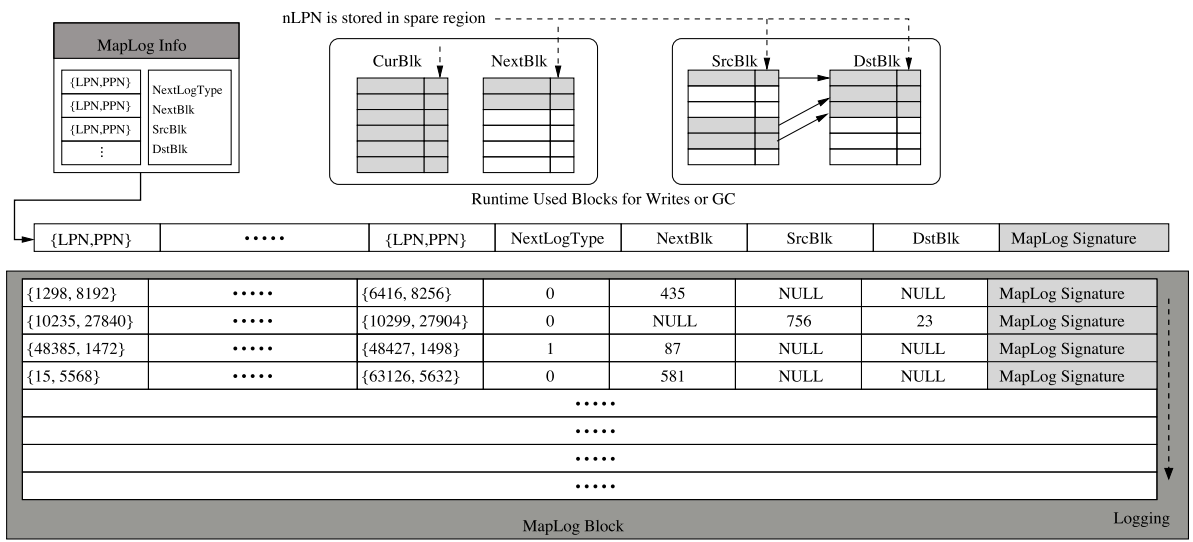

Fig. 2. Runtime Metadata update and logging.

whenever write occurs, since writes generate metadata updates. Instead of direct in-NAND update, the metadata updates are logged into in-memory MapLogInfo structure, and when MapLogInfo is filled with some amount of metadata updates, the MapLogInfo is flushed to one page in MapLog block. The logging trigger points are described in later in this section. In general, there are from 64 to 128 pages in one Block, so from 64 to 128 MapLogInfos can be logged in one MapLog Block.

The log management scheme is depicted in Figure 2. As shown in the figure, the MapLogInfo is logged to MapLog block from start to end of the block. When MapLog Block is full filled with MapLogInfo pages, there is no space for logging. Then, we need to make available log region. Before making available MapLog block, the in-memory metadata is synced to NAND flash memory, and these are considered as up-to-date snapshot of in-NAND metadata information. After the syncing of in-memory metadata information to NAND flash, one free block is selected for MapLog block. And After that, logging mechanism is repeated. On the other hand, when in-memory metadata is synced to NAND flash, Rootinfo also should be updated containing locations of new in-NAND meta blocks, since the snapshot is also written to free region. Rootinfo is also written to one page in Root Block, as logging manner.

The MapLogInfo data structure is used for aggregation of some amount of mapping changes. At some point of time, that is data block changes or GC triggers, the MapLogInfo is written to MapLog block, and then another MapLogInfo is created for later logging of metadata changes. The MapLogInfo data structure consists of array of mapping pair for logical address and corresponding physical address, which represents mapping changes for the current logging. In addition to that, MapLogInfo has information for next log block, NextLogType and (NextBlk, or SrcBlk and DstBlk). The NextLogType indicates type of next log, not current. The NextBlk indicates next data block for write requests. The SrcBlk and DstBlk are source and destination block for GC operation. The information for next log is used for recovery which was not logged in MapLog block. There are two types of data block changes; The one is for block changes during real data writes and 


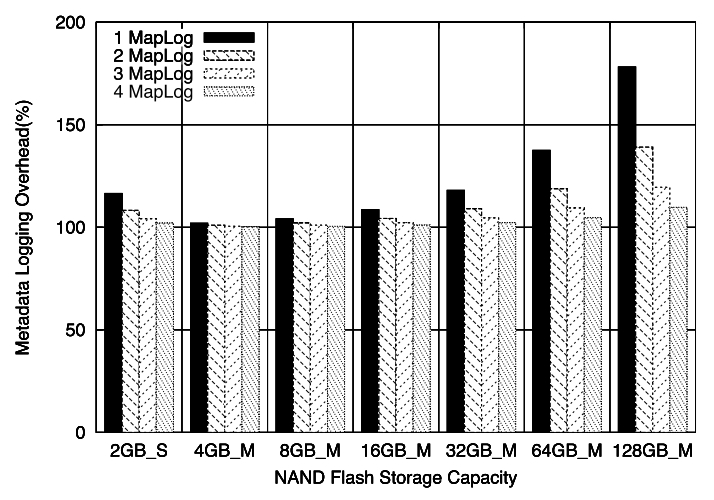

Fig. 3. PLoggging Overhead for full random requests.

Table I. The average portions of scan region during PLR.

\begin{tabular}{|r||c|c|c|c|}
\hline Capacity & 1 MapLog & 2 MapLog & 3 MapLog & 4 Maplog \\
\hline \hline 2GB_S & 0.076 & 0.083 & 0.095 & 0.119 \\
\hline 4GB_M & 0.055 & 0.067 & 0.092 & 0.114 \\
\hline 8GB_M & 0.044 & 0.05 & 0.063 & 0.087 \\
\hline 16GB_M & 0.038 & 0.043 & 0.049 & 0.061 \\
\hline 32GB_M & 0.038 & 0.04 & 0.043 & 0.049 \\
\hline 64GB_M & 0.038 & 0.039 & 0.04 & 0.043 \\
\hline 128GB_M & 0.039 & 0.039 & 0.04 & 0.042 \\
\hline
\end{tabular}

the other is for block chages due to GC. Accordingly, there are two types of logs. The NextLogType indicates the type of next log. Thus, when the next writing block is changes, according Map log is flushed into MapLog block.

The logging method is as follows. Whenever write occurs, the mapping changes are aggregated in main-memory MapLogInfo as array of mapping pair with following notation, (nLPN, nPPN). Also, the $\mathrm{nLPN}$, is saved in the spare region of corresponding physical page, which is important for power loss recovery, that was not logged due to abrupt power loss. The mapping changes are recorded until all the pages of the current data block exhausted. When all the pages of the current block are exhausted, the logging of mapping changes is done, and the remaining job is to set the next log information. Since a new block is required for incoming write request, another free block should be selected from free block pool. If a block can be selected, the selected block number is recorded in NextBlk field within MapLogInfo structure. However, if free blocks are not enough, certain block should be reclaimed to be erased, that is GC. In this case, the selected $S r c B l k$ and DstBlk is recorded in MapLogInfo. Then the MapLogInfo is logged into MapLog Block.

The PLR steps are as follows. At the first step, FTL tries to find the latest RootInfo by scanning Root block. In the second step, FTL recovers the metadata snapshot that consists of BlkInfo[], BlkInfoList[], and page mapping table by retrieving BlockMap and PageMap blocks. In the third step, the metadata changes that are saved in the MapLog block are recovered. In the last step, metadata changes that were not logged in MapLog block are recovered, which can be done by scanning, so called Next Blk, the next $\log$ block information is stored in last MapLogInfo in MapLog Block. The Next Blk can be data block or GC block. The logical address of the block can be achieved by retrieving spare region of each page. 


\section{Evaluation}

The metadata logging and recovery scheme is implemented as a type of device driver within Linux MTD layer. For the evaluation, we have setup simulation environment with simple embedded system evaluation board and NAND simulator. The physical characteristics of NAND flash is referenced from Samsung datasheet [6]. In our design, there are two metadata logging overhead, the one is logging metadata changes in MapLog block, and the other is flushing the snapshot of metadata. In the evaluation, we estimate the additional overhead that incurs during storage system usage. We generate full random write requests and estimate the metadata logging overhead as storage capacity increases from $2 \mathrm{~GB}$ to $128 \mathrm{~GB}$. The estimated overhead is described in Figure 3. In the figure, y-axis represents metadata logging overhead, which is the normalized amount of write for flash systems. From the results, we identify that the overhead is about $70 \%$ for 1 MapLog block with $128 \mathrm{~GB}$ flash memory. The reason for hugh overhead is frequent flushing for BlockMap and PageMap snapshot. The amount of snapshot is proportional to the storage capacity, and the frequency for flushing snapshot is reverse proportional to the number of MapLog block. The overheads are dramatically downs as the number of MapLog block increases. Thus, FTL had better to have at least three or four MapLog blocks. For all cases, the metadata logging overhead is less than $9 \%$, which means overall write performance is degraded less than $9 \%$ if the FTL uses four MapLog blocks.

The recovery scheme is evaluated with the same environment. For each storage configuration, the scanning area and recovery time after abrupt power loss are evaluated as number of MapLog blocks varies from one to four. The estimated scanning area are summarized in Table I. As shown in the table, the absolute recovery coverage is extremely limited by small scanning area in comparison with huge capacity. For all cases, the recovery coverage is estimated less than 0.1 percent. The proper number of MapLog blocks has good effect on the proposed metadata logging and recovery scheme.

\section{Conclusions}

The designed recovery scheme is based on the page-level mapping FTL. In the designed FTL, metadata blocks includes, Root block, BlockMap block, PageMap block, and MapLog block. All the metadata changes for the currently used data block are aggregated in main memory, and then are logged into one page of dedicated flash block, MapLog. By doing this, all the metadata changes can be logged into dedicated block, which minimize metadata update frequency. At the recovery stage, only metadata blocks are retrieved. The designed FTL metadata logging and recovery scheme support fast sudden power loss recovery, with small amount of metadata logging overhead.

\section{Acknowledgments}

The Research was supported by the NRF grant funded by the MEST (No. 2010-0021094). 